\title{
On the construction of smooth ergodic skew-products $\dagger$
}

\author{
MAHESH G. NERURKAR $\ddagger$ \\ Department of Mathematics, The Ohio State University, Columbus, Ohio 43210, USA
}

(Received 10 January 1986 and revised 10 October 1987)

\begin{abstract}
In this paper we prove results about lifting dynamical and ergodic properties of a given smooth dynamical system to its skew-product extensions by smooth cocycles. The classical small divisor argument shows that in general such results are not possible. However, using the notion of the 'fast periodic approximation' introduced by A. Katok, we will show that if the dynamical system admits such a 'fast periodic approximation' then indeed a certain qualitative behaviour which is prohibited by small divisor type conditions is now in fact generic. The techniques are also applied to show that 'recurrent-proximal' behaviour of solutions of linear differential equations with almost periodic coefficients is generic under suitable conditions on the coefficient matrix.
\end{abstract}

\section{Introduction and basic definitions}

1.1 .

Let $\Omega$ be a compact, connected $C^{\infty}$ manifold. Let $T$ denote either the group of integers $(\mathbb{Z})$ or reals $\left((\mathbb{R})\right.$. A triple $(\Omega, T, \mu)$ is called a $\left(C^{k}\right.$-smooth $)$ dynamical system if $T$ acts on $\Omega$ with a jointly $C^{k}$ (i.e. $k$ times continuously differentiable $(k \in \mathbb{N} \cup\{\infty\}$ ) action $(w, t) \rightarrow w \cdot t, \forall w \in \Omega, t \in T$ and preserving a smooth Borel probability measure $\mu$ on $\Omega$. If $T=\mathbb{Z}$, we will denote again by letter $T$ the diffeomorphism generating the $\mathbb{Z}$ action and if $T=\mathbb{R}$ we will denote by $\left(T_{t}\right)_{t \in \mathbb{R}}$ the one parameter group of diffeomorphisms of $\Omega$.

Given a connected Lie group $G$, a cocycle from $(\Omega, T)$ into $G$ is a continuous map $\phi: \Omega \times T \rightarrow G$ such that $\phi\left(w, t_{1}+t_{2}\right)=\phi\left(w \cdot t_{1}, t_{2}\right) \phi\left(w, t_{1}\right), \forall w \in \Omega, t_{1}, t_{1} \in T$. A cocycle $\phi$ is of class $C^{k}$ if for each $t \in T$ the map $w \rightarrow \phi(w, t)$ is a $C^{k}$ map. Let $Z_{k}(\Omega, G)$ denote the set of all $C^{k}$ cocycles into $G$. If $T=\mathbb{Z}$, the cocycle $\phi$ is completely determined by its values on $\Omega \times\{1\}$ and hence will be identified with a function on $\Omega$ into $G$, (i.e. cocycle $\phi$ determined by a function $\phi$ is given by $\phi(w, n)=\phi\left(T^{n-1} w\right) \phi\left(\cdots \phi(w), n \in \mathbb{Z}^{+}\right)$. Thus for $T=\mathbb{Z}$, we will identify $Z_{k}(\Omega, G)$ with $C^{k}(\Omega, G)$ the space of $C^{k}$ functions from $\Omega$ into $G$. Thus consequently the usual $C^{k}$ metric on $C^{k}(\Omega, G)$ induces a metric $D_{k}$ on $Z_{k}(\Omega, G)$. We denote by \|\|$_{k}$ the $C^{k}$ norm on $C^{k}(\Omega, \mathbb{R})$. Metrics on all other spaces will be denoted by the letter $d$. 
Suppose $Z$ is another $C^{\infty}$ manifold on which a Lie group $G$ acts on the left with a jointly $C^{k}$ action $(g, z) \rightarrow g z$. Then given a cocycle $\phi \in Z_{k}(\Omega, G)$, we define on $Z \times \Omega$ the skew product T-action by setting $(z, w) \cdot t=(\phi(w, t) z, w \cdot t)$. In the case $T=\mathbb{Z}$, the diffeomorphism generating the skew-product action will be denoted by $T_{\phi}$, i.e. $T_{\phi}(z, w)=(\phi(w) z, T w)$. If $\nu$ is a $G$ invariant Borel measure on $Z$ then clearly $\nu \times \mu$ is invariant under the skew-product action. The following important examples of this set up should be kept in mind. ( $a$ ) Let $Z=G$ and $G$ acts on itself by left multiplication. Here $\nu=\eta-a$ left Haar measure on $G$, is an invariant measure. (b) Let $G=G L(n, \mathbb{R})$ - the general linear group and $Z=p^{n-1}(\mathbb{R})$ - the real projective $n-1$ space. In this case we do not have any invariant measure on $Z$.

Given any $f \in C^{k}(\Omega, G)$, it generates a cocycle $1^{f} \in Z_{k}(\Omega, G)$ by setting $1^{f}(w, t)=$ $f(w \cdot t) f(w)^{-1}$. Cocycles of this form are called coboundaries and the set of all such coboundaries will be denoted $B_{k}(\Omega, G)$. The trivial cocycle is the cocycle $1^{1}$, generated by the map $w \rightarrow e$ (-the identity element of $G$ ) and will be denoted by 1 . Given a $\phi \in Z_{k}(\Omega, G)$ and $1^{f} \in B_{k}(\Omega, G)$ set, $\phi \cdot 1^{f}(w, t)=f(w \cdot t) \phi(w, t) f(w)^{-1}$. It is easy to verify that $\phi \cdot 1^{f} \in Z_{k}(\Omega, G)$. Given $\phi_{1}, \phi_{2} \in Z_{k}(\Omega, G)$ we call them cohomologous via a transfer function $f$ if $\phi_{2}=\phi_{1} \cdot 1^{f}$ for some $f \in C^{k}(\Omega, G)$. Cohomologous cocycles gives rise to isomorphic skew-product actions.

Our main goal is to construct cocycles for which the corresponding skew-products are ergodic. The following property of the action of $G$ on $Z$ is necessary for the ergodicity of the skew-product actions, (see [6], also refer to [22] for a similar result). Let $\nu$ be a $\sigma$-finite $G$ invariant measure on $Z$, then the triple $(Z, G, \nu)$ is said to have the $L^{\infty}$-fixed point property if every $G$ invariant weakly compact set $K$ contained in the closed unit ball of $L^{\infty}(Z, \nu)$ contains a fixed point for the $G$ action. This property also helps in constructing cocycles with ergodic skew-products. We list a few examples of actions having this property. (1) If $\nu$ is a finite Borel measure then $(Z, G, \nu)$ has this property. (2) Let $G$ be amenable, $Z=G$ and the action of $G$ be by left-multiplication. Take $\nu=\eta-a$ left Haar measure. This system does have the $L^{\infty}$ fixed point property. (3) Let $Z=\mathbb{R}^{2}$ and $G=S L(2, \mathbb{R})$ with standard linear action on $Z$ and $\nu$ be the Lebesque measure on $\mathbb{R}^{2}$. The $(Z, G, \nu)$ has the $L^{x}$ fixed point property.

\subsection{Lifting ergodicity by smooth cocycles}

One important question in the study of skew-product dynamical systems has been lifting ergodicity i.e. if $(\Omega, T, \mu)$ is ergodic, can one find a cocycle $\phi$ such that the corresponding skew-product flow is ergodic with respect to the product measure? If in the above set up everything is just 'continuous' (and not smooth) then the existence of a continuous cocycle $\phi$ giving ergodic skew-products is known - even for more general dynamical systems, where $T$ can be any reasonable amenable group, (see [18]). In fact such cocycles are residual in the class $\bar{B}_{0}(\Omega, G)$, (we will denote by $\bar{B}_{k}$ the closure of $B_{k}$ in $Z_{k}$ ). However even for integer actions (i.e. $T=\mathbb{Z}$ ) the problem of producing sufficiently smooth function $\phi$ for which $T_{\phi}$ is ergodic is delicate and difficult. In fact the classical 'small divisor' argument shows that for some dynamical systems this is impossible. For example, let $\Omega=S^{1}$ - the circle. 
$T=R_{\alpha}$ - the irrational rotation by $\alpha$, where $\alpha$ is badly approximable by rationals. Then given any smooth enough (say $C^{k}, k \geq 2$ ) function $\phi: \Omega \rightarrow \mathbb{R}$ with $\int_{\Omega} \phi d m=0$ ( $m$ being the Lebesque measure on $S^{1}$ ), the functional equation $\phi(w)=$ $f\left(R_{\alpha} w\right)-f(w)$ has always a solution $f$ of class - say $C^{k-2}$. This result of KolmogorovSiegel shows that $\bar{B}_{k}\left(S^{1}, \mathbb{R}\right) \subseteq B_{k-2}\left(S^{1}, \mathbb{R}\right)$ and hence $\forall_{\phi} \in \bar{B}_{k}(\Omega, \mathbb{R})$ the skew-product diffeomorphism $\left(R_{\alpha}\right)_{\phi}$ on $\mathbb{R} \times S^{1}$ can not be ergodic.

A very different phenomenon occurs when $T$ is a point transitive Anosov diffeomorphism. In this case A. Livshitz has given (see [16]) a precise condition for the solvability of the cohomology equation $f(T w)-f(w)=\phi(w)$, for a given smooth $\phi$. Using this, one can show that $\bar{B}_{1}(\Omega, \mathbb{R})=B_{1}(\Omega, \mathbb{R})$ and hence lifting ergodicity in the class $\bar{B}_{1}(\Omega, \mathbb{R})$ is impossible. However using a Parry-Jones type argument along with $A$. Livshitz's result one can show that cocycles lifting ergodicity in the class $Z_{1}(\Omega, G)$ are residual when $G=\mathbb{V}^{n}$ - the $n$ torus. A similar result about density of cocycles lifting ergodicity in the class $Z_{1}(\Omega, G)$ for any compact connected Lie group $G$ is obtained by M. Brin (see [2]). We also remark that this result of A. Livshitz has been generalized to the case when $T$ is a point transitive diffeomorphism satisfying a 'closing lemma', (see [12]). Also versions of this result for geodesic flows are known. Now a result of J. Hawkins, ([5]) based on slight modification of Parry-Jones argument shows that ergodicity can be generically lifted in the class $Z_{k}(\Omega, G)$ for any diffeomorphism if $G=\mathbb{J}^{n}-$ the $n$-torus. This shows that lifting ergodicity in the class $Z_{k}$ and in the class $\bar{B}_{k}$ are in some sense different problems. Also non compactness and non abelianness of the group $G$ contribute substantially to the difficulties in constructing smooth cocycles lifting ergodicity.

In this paper we prove that the set of cocycles which yield the ergodic skew-product is a residual set in $\bar{B}_{k}$, provided the transformation $T$ admits 'a fast periodic approximation'. We develop a smooth version of a technique of Glasner and Weiss, (see [4]). The small divisor argument shows that in general their technique has no smooth analog. The essence of their technique is the construction of a coboundary $C^{0}$ with certain 'desired properties' which also is close to the identity cocycle in $C^{0}$ norm. Replacing the closeness in $C^{0}$ norm by the one in $C^{k}$ norm is in general impossible and this is the main obstacle. We will show that if the transformation admits 'a fast periodic approximation' with 'sufficient speed' then this obstacle can be overcome.

\subsection{Linear system of differential equations}

In the case of a flow, besides lifting ergodicity, one also wants to make certain skew-product extensions 'proximal'. This notion is of importance in the study of the qualitative behaviour of a linear differential system. By a linear differential system we mean a family of linear differential equations given by,

$$
\dot{x}=A(\omega \cdot t) x, \quad x \in \mathbb{R}^{n}, \quad \omega \in \Omega, \text {. }
$$

where $\left(\Omega, T_{t}\right)$ is a $C^{k}$ flow and $A: \Omega \rightarrow M(n, \mathbb{R})$ is a continuous map, $(M(n, \mathbb{R})$ is the set of $n \times n$ real matrices). We will assume that $\Omega$ is a compact, connected $C^{\infty}$ manifold and the flow will always be minimal and almost-periodic, (i.e. the family 
of maps $\left\{\omega \rightarrow T_{t} \omega \mid t \in \mathbb{R}\right\}$ is equicontinuous). For example let $\dot{x}=A(t) x$ be a single differential equation where the entries of the matrix $A(t)$ are (Bohr) almost-periodic functions. Let $\Omega$ be the hull of $A(t)$. If $\Omega$ is a $C^{\infty}$ manifold, this problem gets transformed into our'general set up.

Let $X_{A}(w, t): \Omega \times \mathbb{R} \rightarrow G L(n, \mathbb{R})$ be the fundamental matrix solution of $(I)$ satisfying $X_{A}(w, 0)=I$ - the identity matrix, $\forall \omega \in \Omega$. Then $X_{A}$ is a cocycle. Also $A(w)$ can be recovered from $X_{A}$ (i.e. $\left.A(w \cdot t)=\left[(d / d t) X_{A}(w, t)\right] X_{A}(w, t)^{-1}\right)$. In this way we shall here onwards identify the set $Z_{r}(\Omega, G L(n, \mathbb{R}))$ with systems $\dot{x}=A(w \cdot t) x$. Furthermore note that $X_{A}$ and $X_{B}$ are cohomologous iff systems $\dot{x}=A(w \cdot t) x$ and $\dot{x}=B(w \cdot t) x$ are kinematically similar.

Consider $\sum=p^{n-1} \times \Omega$ (where $p^{n-1}$ is the real projective space) and the skewproduct flow on $\sum$ defined by the cocycle $X_{A}$ i.e. $([x], w) \cdot t=\left(\left[X_{A}(w, t) x\right], w \cdot t\right)$ where $[x]$ denotes the ray containing $x \in \mathbb{R}^{n}$. System $(I)$ will be called recurrent if the skew-product flow on $\sum$ is minimal. System $(I)$ is said to be proximal if the projection $\pi: \sum \rightarrow \Omega(\pi([x], w)=w)$ is a proximal extension, i.e. given $\left(\left[x_{1}\right], w_{0}\right)$, $\left(\left[x_{2}\right], w_{0}\right) \in \sum$ there exists a sequence $t_{n} \in \mathbb{R}$ such that,

$$
d\left(\left(\left[x_{1}\right], w_{0}\right) \cdot t_{n},\left(\left[x_{2}\right], w_{0}\right) \cdot t_{n}\right) \rightarrow 0 \quad \text { as } n \rightarrow \infty .
$$

Qualitatively this means that for any $w \in \Omega$, the angle between any two solutions of (I) tends to 0 for some sequence $t_{n}$ of times.

It is important to know when system $(I)$ is both recurrent and proximal. For example if $A(\omega) \equiv A$ is a constant matrix, this will never happen. This is because recurrence requires that eigenvalues of $A$ be purely imaginary, (real eigenvectors will give rise to proper closed invariant sets for the skew-product flow), but then in this case the flow on $\sum$ preserves distances between points, and hence can not be proximal. Note that in this case $X_{A}(\omega, t)=e^{t A}$, following A. Katok cocycles of this type (namely homomorphism from $\mathbb{R}$ to $G L(n, \mathbb{R})$ ) will be called constant cocycles. Now suppose that the flow on $\Omega$ is periodic (but $A$ is not necessarily constant) then the classical Floquet theorem says that in this case $X_{A}$ is cohomologous to some constant cocycle. This shows that recurrent-proximal behaviour is impossible when $\left(\Omega, T_{t}\right)$ is a periodic flow. We shall show that if the flow $\left(\Omega, T_{t}\right)$ is almost periodic (but not periodic) and admits 'fast periodic approximation' then recurrent-proximal behaviour is generic. In passing we mention that under similar hypothesis A. Katok has shown the existence of cocycles not cohomologous to any constant cocycle [12].

\section{Fast periodic approximation and statement of the main theorem}

Definition 2.1. Consider a $C^{r}$-smooth discrete dynamical system $(\Omega, T, \mu)$ on a $C^{\infty}$ manifold $\Omega$. Let $a(n) \geq 0, n \in \mathbb{N}$ be a sequence such that $\lim _{n \rightarrow \infty} a(n)=0$.

Following A. Katok [12], the diffeomorphism $T$ is said to admit a $C^{r}$-fast-periodic approximation (or $C^{r}$-rigid) with speed $a(n)$ if there exists a sequence $\left(q_{n}\right)_{n \in \mathbb{N}}$ of positive integers and a consant $K>0$ such that $q_{n} \rightarrow \infty$ and,

$$
\left\|h \circ T^{q_{n}}-h\right\|_{r} \leq K\|h\|_{r+1} a\left(q_{n}\right), \quad \forall n \in \mathbb{N}, \quad \forall h \in C^{r+1}(\Omega, \mathbb{R}) .
$$


If $T$ is $C^{\infty}$ and above inequality holds $\forall r \in \mathbb{N}$, (with $K$ depending on $r$ ) then we say $T$ is $C^{\infty}$ rigid with speed $a(n)$.

The following is a typical example of such diffeomorphisms.

Example. Let $\Omega=S^{1}$, be the circle and $T=R_{\alpha}$, be the irrational rotation by $\alpha$. If $\exists a$ sequence $\left(p_{n} / q_{n}\right)_{n \in \mathbb{N}}$ of irreducible fractions with $q_{n} \rightarrow \infty$ as $n \rightarrow \infty$ and constants $K>0, p \in \mathbb{N}$ and $0<\varepsilon<1$ such that

$$
\left|\alpha-p_{n} / q_{n}\right|<K /\left(q_{n}\right)^{p+1+\varepsilon}
$$

then $\left(\Omega, R_{\alpha}\right)$ is $C^{\infty}$ rigid with speed $a(n)=1 / n^{p+\varepsilon}$. If $\alpha$ is a Liouville number i.e. $\left|\alpha q_{n}-p_{n}\right|<K /\left(q_{n}\right)^{n}$ for some sequence $p_{n}, q_{n}$ of integers with $q_{n} \rightarrow \infty$ and $K>0$ is a constant then we can take $a(n)=1 / n^{n}$.

An immediate generalization of this example is obtained by taking $\Omega=\mathbb{t}^{n}$ - the $n$-torus and $R_{\alpha}$ - the irrational rotation by $\bar{\alpha}=\left(\alpha_{1}, \ldots, \alpha_{n}\right)$, where $\bar{\alpha}$ is a 'Liouville vector' i.e. $\alpha_{1}, \ldots, \alpha_{n}$ are rationally independent irrationals and for some sequence $q_{n}$ of integers such that $q_{n} \rightarrow \infty$ as $n \rightarrow \infty$ one has

$$
\left\|\alpha_{i} q_{n}\right\| \leq K /\left(q_{n}\right)^{n}, \quad \forall i, n,
$$

where $K>0$ is a constant and \|\|$\|$ denotes the distance from the nearest integer. Again $\left(\Omega, R_{\bar{\alpha}}\right)$ is $C^{\infty}$ rigid with speed $\left(1 / n^{n}\right)$.

We also mention that besides this prime example, examples constructed by Anosov and Katok in [1] also admit $C^{r}$-fast periodic approximations.

Now we state our main theorem.

THEOREM 2.2. Let $(\Omega, T, \mu)$ be a $C^{r}$ dynamical system with a smooth (i.e. with positive $C^{\infty}$ density) invariant ergodic probability measure $\mu$. Let $(\Omega, T)$ admit $C^{r}$ fast-periodic approximation with speed $a(n)$. Let $G$ be a connected Lie group acting $C^{r}$-smoothly on a connected $C^{\infty}$ manifold $Z$ and preserving a $\sigma$-finite ergodic measure $\nu$. Assume that $(Z, G, \nu)$ has the $L^{\infty}$ fixed point property. Consider the following conditions (A) and $(\mathrm{B})$

Condition (A). (Uniform boundedness of derivatives). There exists a constant $C>0$ such that

$$
\left\|D^{r} T^{n}\right\|_{0}<C, \quad \forall n \in \mathbb{Z},
$$

where $D^{r} T^{n}$ is the $r$ th derivative of $T^{n}$ and \|\|$_{0}$ is the sup norm.

Condition (B). ( $C^{0}$ rigidity). There exists a constant $K>0$ such that $\left\|h \circ T^{q_{N}}-h\right\|_{0} \leq$ $K\|h\|_{0} a\left(q_{N}\right), \forall h \in C(\Omega, \mathbb{R})$.

(I) Suppose $T$ satisfies condition (A) and $a(n)=1 / n^{4 r+1+\rho},(0<\rho<1)$ then the set $\left\{\phi \in \bar{B}_{r}(\Omega, G) \mid\left(Z \times \Omega, T_{\phi}, \nu \times \mu\right)\right.$ is ergodic $\}$ is residual in $\bar{B}_{r}(\Omega, G)$.

(II) Suppose $T$ satisfies condition (B) and $a(n)=1 / n^{2 r+1+\rho},(0<\rho<1)$ then we have the same conclusion as above.

Suppose $G=\mathbb{R}$ then one may only assume $a(n)=1 / n^{2 r+1+e}$ or $a(n)=1 / n^{r+1+\rho}$ depending respectively on whether condition (A) or (B) holds.

(III) Suppose $T$ satifies either condition (A) or (B) and $a(n)=1 / a^{n},(a>1$ is a constant $)$. Then the same residuality result holds in the class $\bar{B}_{\infty}(\Omega, G)$. 
(IV) Suppose $G=\mathbb{R}$ and $(\Omega, T)$ be the irrational rotation on circle. Let the rotation number $\alpha$ satisfy, the condition $\left|\alpha-p_{n} / q_{n}\right|<K / q_{n}^{r+1+\rho},(0<\rho<1)$ i.e., $a(n)=$ $1 / n^{r+\rho}$ (see the example). Then the same residuality result holds in the class $\overline{B_{r}(\Omega, \mathbb{R})}$.

Remark 2.3. (a) For the example of irrational rotation on the circle, (IV) above gives us exactly the generic version of a theorem of Krygin [15]. We also believe that for $G=\mathbb{R}$, the 'least speed' one needs to get a generic lifting of ergodicity in the class $\bar{B}_{r}$ is $a(n)=1 / n^{r+p}, 0<\rho<1$.

(b) If $\mu$ is the unique ergodic measure on $\Omega$ and either (i) $G$ is amenable or (ii) $G=S L(n, \mathbb{R})$ and $Z=P^{n-1}(\mathbb{R})$ then in the conclusion of theorem (2.2) ergodicity can be replaced by unique ergodicity.

(c) Although we do not discuss 'affine cocycles' in this paper, using the techniques developed here, we can prove an 'affine extension' of theorem (2.2). See [14] for a continuous version. As a consequence we get the following theorem.

TheOREM 2.4. Let $(\Omega, T, \mu)$ be as in theorem (2.2). Let $G=\mathbb{T}^{n}$ be the $n$-torus and $\sigma$ be any automorphism of $G$. Then the set $\left\{\phi \in C^{r}(\Omega, G) \mid\right.$ the map $T_{\phi}(g, w)=$ $(\sigma(\phi(w) g), T w)$ on $G \times \Omega$ is ergodic with respect to the product measure $\}$ is residual in $C^{r}(\Omega, G)$, (here if $T$ satisfies (III) of theorem (2.2)), then take $r=\infty$ ).

Finally we state our result on lifting minimality and getting proximal extension in the context of linear differential systems. The notion of fast periodic approximation is similar, the diffeomorphism $T^{q_{n}}$ in the definition (2.1) is replaced by $T_{q_{n}}$ where $\left(T_{t}\right)_{t \in \mathbb{B}}$ is the one parameter group of diffeomorphisms generated by the flow. A typical example of such a flow is again the flow on the $n$-torus generated by irrational rotation by Liouville vectors. For simplicity we state the following theorem only for such systems. Also we remark that here that the topology on $Z_{k}(\Omega, S L(n, \mathbb{R}))$ is given by the metric

$$
D_{k}\left(\phi_{1}, \phi_{2}\right)=\operatorname{Sup}_{0<t \leq 1} D_{k}\left(\phi_{1}(\cdot, t), \phi_{2}(\cdot, t)\right),
$$

where $\phi_{1}, \phi_{2} \in Z_{k}(\Omega, S L(n, \mathbb{R}))$.

THEOREM 2.5. Let $\left(\Omega, T_{1}\right)$ be the rotation flow on the n-torus generated by a Liouville vector. Then the set

$$
\left\{X_{A} \in \bar{B}_{x}(\Omega, S L(n, \mathbb{R})) / \dot{x}=A(\omega \cdot t) x\right.
$$

is recurrent and proximal $\}$ is residual in $\bar{B}_{x}(\Omega, S L(n, \mathbb{R}))$.

2. Proof of theorem 2.2. Let $H=L^{1}(Z \times \Omega, \nu \times \mu)$ and

$$
H_{0}=\left\{f \mid f \in H, \int_{Z x \Omega} f d \nu x \mu=0\right\} .
$$

Let \|\| be the $L^{1}$ norm on $H$. Given a cocycle $\phi \in Z_{r}(\Omega, G)$, define the operator $U_{\phi}$ on $H$ by setting $U_{\phi} f(z, w)=f(\phi(w) z, T w)$. Let

$$
V_{\phi}^{N}=\frac{1}{N} \sum_{i=0}^{N-1}\left(U_{\phi}\right)^{i}
$$


Also, given a function $\psi \in C^{r}(\Omega, G)$, define the operator $L_{\psi}$ on $H$ by $L_{\psi} f(z, w)=$ $f(\psi(w) z, w)$.

Now given $f \in H_{0}, \varepsilon>0$ and $m \in \mathbb{N}$, define $W(f, \varepsilon, m)=\left\{\phi \mid \phi \in \bar{B}_{r}(\Omega, G)\right.$ such that $\exists M \in \mathbb{N}, M>m$ and $\left.\left\|V_{\phi}^{M} f\right\|<\varepsilon\right\}$. Now if

$$
\phi \in \bigcap_{j} \bigcap_{n} \bigcap_{m} W\left(f_{j}, 1 / n, m\right),
$$

(where $\left(f_{j}\right)_{j \in \mathbb{N}} \in H_{0}$ is a dense subset) then $\left(Z \times \Omega, T_{\phi}, \nu \times \mu\right)$ is ergodic, (see [6] for a proof). Thus once we prove that each $W(f, \varepsilon, m)$ is open and dense in $\bar{B}_{r}$ then theorem (2.2) is a consequence of the Baire Category theorem. Openness is easy to verify. To prove density, first observe that $V_{\phi}^{N} L_{\psi}=L_{\psi} V_{\phi .1^{\omega}}^{N}$. This shows that if $1 \in \overline{W(f, \varepsilon, m)}, \forall f, \varepsilon, m$ then $1^{\psi} \in \overline{W(f, \varepsilon, m)}, \forall f, \varepsilon, m, \psi$ i.e. $W(f, \varepsilon, m)$ is dense in $\bar{B}_{r}$. Thus it is enough to prove that, given $f, \varepsilon, m$ and $\delta>0$ there exists a $\psi \in C^{r}(\Omega, G)$ such that, (i) $D_{r}\left(1^{\psi}, 1\right)<\delta$ and $\left\|V_{1^{\prime}}^{M}\right\|<\varepsilon$ for some $M>m$.

Now consider, $\left\|V_{1^{\prime}}^{n} f\right\|=\left\|L_{\psi}^{-1} V_{1}^{n} L_{\psi} f\right\|=\left\|V_{1}^{n} L_{\psi} f\right\|$, (since $L_{\psi}$ is an isometry). Also note that by the ergodic theorem we have,

$$
V_{1}^{n} g(z, y)=\frac{1}{n} \sum_{i=0}^{n-1} g\left(z, T^{i} y\right) \rightarrow \int_{\Omega} g(z, y) d \mu(y) \quad \forall g \in H
$$

where the convergence is in the norm on $H$. Thus

$$
\left\|V_{1^{\omega}}^{n} f\right\| \rightarrow \int f(\psi(w) z, w) d \mu(w) \quad \text { as } \quad n \rightarrow \infty .
$$

Hence if we can choose

$$
\left\|\int_{\Omega} f(\psi(w) z, w) d \mu(w)\right\|<\varepsilon / 2,
$$

then by choosing $M$ large enough, we can make sure that $\left\|V_{1}^{M} f\right\|<\varepsilon$. Thus we have reduced the proof of the theorem to the following lemma.

Lemma 1. Given $f \in H_{0}, \varepsilon<0$ and $\delta>0$, there exist $a \psi \in C^{r}(\Omega, G)$ such that (i) $D_{r}\left(1^{\psi} .1\right)<\delta$ and (ii) $\left\|\int_{\Omega} f(\psi(w) z, w) d \mu(w)\right\|<\varepsilon$.

Proof. Let $0<\gamma^{\prime}<\frac{1}{2}$ be a small number (it's relation to $\varepsilon$ will be determined later). Since $C_{c}(Z \times \Omega)$ is dense in $L^{1}(Z \times \Omega)$, standard approximation arguments allow us to assume (without loss of generality) that the given $f$ is continuous, has compact support and $\|f\|<\varepsilon / 8$. We will first assume that $f$ depends on $z$ alone, i.e. $f(z, w) \equiv$ $f(z)$ and let $M_{1}=\sup \{|f(z)| / z \in Z\}$.

We will fix a family $\left\{\left(0_{\alpha}, \phi_{\alpha}\right) \mid 1 \leq \alpha \leq R\right\}$ of local charts so that (i) $\left(0_{\alpha}\right)_{\alpha=1}^{R}$ is an open cover of $\Omega$

(ii) $\phi_{\alpha}: 0_{\alpha} \rightarrow Q$ (where $Q \equiv\left\{\bar{x}=\left(x_{1}, \ldots, x_{n}\right) \in \mathbb{R}^{n} / 0<x_{i}<1\right\}, n=\operatorname{dim} \Omega$ ) is a $C^{x}$ diffeomorphism such that $\left(\phi_{\alpha}\right)_{*}\left(\left.\mu\right|_{0_{\alpha}}\right)=\left.m\right|_{Q}$, where $\left.\mu\right|_{0_{\alpha}}$ and $\left.m\right|_{Q}$ are the normalized restrictions of measure $\mu$ and the Lebesque measure $m$ to $0_{\alpha}$ and $Q$ respectively, (such a chart can be chosen because $\mu$ has a positive $C^{\infty}$ density in each chart, see [1]). Let $M_{2}=\sup _{1<\alpha<R}\left\|\phi_{\alpha}\right\|_{r+1}$. Set $M=\max \left\{M_{1}, M_{2}\right\}$, rote that $M$ depends only on $r, f$ and the manifold $\Omega$.

Since $(Z, G, \nu)$ has the $L^{\infty}$ fixed point property, there are $c_{i} \in[0,1]$ and $g_{i} \in G$, $1 \leq i \leq s$ such that $\sum_{i=1}^{s} c_{i}=1$ and $\left\|\sum_{i=1}^{s} c_{i} f_{g_{i}}\right\|<\varepsilon / 4$, where $f_{g_{i}}(z)=f\left(g_{i} z\right)$, (a sketch 
of the proof of this fact will be provided at the end). Divide interval $[0,1]$ into subintervals $\Lambda_{j}$ of length $c_{j}$ as shown in the figure.

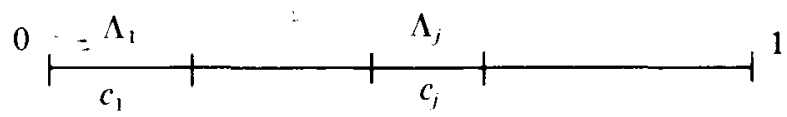

Define a $C^{\infty}$ map $h:[0,1] \rightarrow G$ by setting it equal to $g_{i}$ on 'most of the interval $\Lambda_{i}$ ' and making sure that,

$$
\left\|\int_{0}^{1} f_{h(t)}(z) d t\right\|<\varepsilon / 2 .
$$

Now onwards let assumptions in (I) of theorem (2.2) hold. Pick $N \in \mathbb{N}$ such that,

$$
\frac{M}{q_{N}}<\frac{\varepsilon}{10} \quad \text { and (ii) } \frac{L K(\hat{M})^{2}(2)^{2 r+1}}{\left(q_{N}\right)^{p}}<\delta,
$$

where $L$ and $\hat{M}$ are constants depending only on $r, \varepsilon$, functions $f, h$, the map $T$ and the manifold $\Omega$ (they will be explicitly described later).

Using Rokhlin's lemma pick a Borel set $E \subseteq \Omega$ such that (i) $E, T E, \ldots, T^{\left(q_{N}\right)^{2}-1} E$ are mutually disjoint and

$$
\mu\left(\bigcup_{i=0}^{\left(q_{N}\right)^{2}-1} T^{i} E\right)>1-\left(\gamma^{\prime} / M\right)
$$

Let $\left(B_{j}\right)_{j=1}^{p}$ be a partition of $E$ into disjoint Borel sets with positive measure such that for each $1 \leq i \leq p,(p \leq R)$ there is some $\alpha(i) \in\{1,2, \ldots, R\}$ such that $B_{i} \subseteq 0_{\alpha(i)}$. Without loss of generality we assume that each $B_{j}$ is compact, (if not replace it by a compact subset with measure so close to that of $B_{j}$ that (3) is preserved). Let $\xi_{1}>0$ be such that the $\xi_{1}$-neighbourhoods of compact sets $\left\{T^{i} E\right\}_{i=0}^{\left(q_{N}\right)^{2}-1}$ are pairwise disjoint. Now pick $\xi_{2}>0$ such that if $d(x, y)<\xi_{2}$ then

$$
d\left(T^{i} x, T^{i} y\right)<\xi_{1} \quad \forall 0 \leq i \leq\left(q_{N}\right)^{2}-1,
$$

set $\xi=\min \left(\xi_{1}, \xi_{2}\right)$. Now pick open sets $V_{j}(1 \leq j \leq p)$ such that, (a) $B_{j} \subseteq V_{j} \subseteq 0_{\alpha(j)} \cap$ $\{\xi$-neighbourhood of $E\}$ (b) $V_{i} \cap V_{j}=\phi, \forall i \neq j$ and (c)

$$
\mu\left(V_{j}-B_{j}\right)<\gamma^{\prime} \mu\left(B_{j}\right) /\left(q_{N}\right)^{2} M .
$$

Note that the sets $\left\{T^{i} V_{j} \mid 1 \leq j \leq p, 0 \leq i \leq\left(q_{N}\right)^{2}-1\right\}$ are pairwise disjoint. Set

$$
V=\bigcup_{j=1}^{p} V_{j}, \quad \lambda=\mu(V) \quad \text { and } \lambda_{j}=\mu\left(V_{j}\right) .
$$

The following sublemma (the proof of which will be given later) is the first step in in the construction of the desired function $\psi$.

Sublemma 2. Let $Q \equiv\left\{\left(x_{1}, \ldots, x_{n}\right) \in \mathbb{R}^{n} / 0<x_{i}<1\right\}, W \subseteq Q$ be an open set, $I \subseteq[0,1]$ be an interval of length $l$ and $f_{\beta}:[0.1] \rightarrow \mathbb{R}, \beta \in J$ be an equicontinuous family of maps. Then given $\varepsilon>0$, and $r \in \mathbb{N}$ there exists a $C^{\infty}$ map $\eta: Q \rightarrow I$ such that,

(a) supp $\eta \subseteq W$ and $\eta \equiv 0$ in a small neighbourhood of $\partial W$ and

$$
\|\eta\|_{i} \leq F\left[\frac{l}{m(W)}\right]^{i}, \quad 1 \leq i \leq r
$$

where $F$ is a constant depending on $r, \varepsilon$, family $f_{\beta}$ and the dimension of $Q$. 
(b)

$$
\left|\frac{1}{m(W)} \int_{W}\left(f_{\beta} \circ \eta\right) d m-\frac{1}{|I|} \int_{I} f_{\beta}(t) d t\right|<\varepsilon, \quad \forall_{\beta} \in J
$$

With the help of above sublemma, we now construct maps $\theta_{j}: V_{j} \rightarrow \mathbb{R}$ as follows:

Let $\theta_{j}=\eta_{j} \circ \phi_{\alpha(j)}$ where $\eta_{j}: \phi_{\alpha(j)}\left(V_{j}\right) \rightarrow I_{j}$ be as in the sublemma where the equicontinuous family of maps is $t \rightarrow f(h(t) z), z \in Z, \varepsilon=\gamma^{\prime}$ and $I_{j}$ is the interval of length $\lambda_{j} / \lambda$ as shown in the figure.

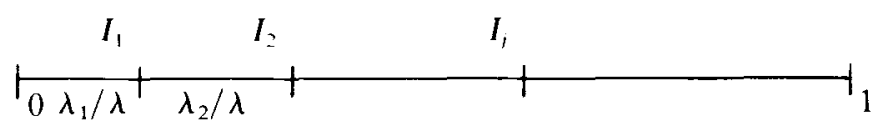

Clearly supp $\theta_{j} \subseteq V_{j}$ and $\theta_{j}$ can be extended to all of $\Omega$ by setting it equal to 0 outside $V_{j}$. Thus $\theta_{j}: \Omega \rightarrow \mathbb{R}$ is $C^{\infty}$ map.

Now

$$
\left\|\theta_{j}\right\|_{r+1}=\left\|\eta_{j} \circ \phi_{\alpha(j)}\right\|_{r+1} \leq A\left\|\phi_{\alpha(j)}\right\|_{r+1}\left\|\eta_{j}\right\|_{r+1},
$$

where $A$ is a constant depending only on $r$, namely it is the number of terms one gets in computing $r+1$ derivatives of composition of two functions.

Thus

$$
\left\|\theta_{j}\right\|_{r+1} \leq A M\left\|\eta_{j}\right\|_{r+1} \leq \frac{A M F\left(\lambda_{j} / \lambda\right)^{r+1}}{m\left(\phi_{\alpha(j)}\left(V_{j}\right)\right)^{r+1}} \quad(\text { by }(5)) .
$$

Now since $\left(\phi_{\alpha}\right)_{*}\left(\left.\mu\right|_{\sigma_{\alpha}}\right)=\left.m\right|_{Q}$, we have

Hence

$$
\frac{\lambda_{j}}{\mu\left(0_{\alpha(j)}\right)}=\frac{\mu\left(V_{j}\right)}{\mu\left(0_{\alpha(j)}\right)}=\frac{m\left(\phi_{\alpha(j)}\left(V_{j}\right)\right)}{m(Q)}=m\left(\phi_{\alpha(j)}\left(V_{j}\right)\right) \text {. }
$$

where

$$
\left\|\theta_{j}\right\|_{r+1} \leq \frac{\tilde{M}}{\lambda^{r+1}}
$$

$$
\tilde{M}=A M F\left[\operatorname{Max}_{1 \leq j \leq R} \mu\left(0_{\alpha(j)}\right)\right]^{r+1} .
$$

Set $\theta^{*}=\sum_{j=1}^{p} \theta_{j}$, then $\theta^{*}$ is a $C^{r+1}$ map with Supp $\theta^{*} \subseteq V$ and since $V_{j}$ 's are disjoint we have,

$$
\left\|\theta^{*}\right\|_{r+1} \leq \tilde{M} / \lambda^{r+1}
$$

First we prove part (I) of theorem (2.2). Define $\tilde{\theta}: \Omega \rightarrow \mathbb{R}$ by settling, and

$$
\begin{aligned}
\tilde{\theta}\left(T^{i} w\right) & =\theta^{*}(w), \forall w \in V, 0 \leq i \leq\left(q_{N}\right)^{2}-1 \\
\tilde{\theta}(w) & =0 \text { outside } \cup\left\{T^{i} V / 0 \leq i \leq\left(q_{N}\right)^{2}-1\right\}
\end{aligned}
$$

Let

$$
\theta(w)=\frac{1}{q_{N}} \sum_{i=0}^{q_{N}-1} \tilde{\theta}\left(T^{i} w\right)
$$

Note that by (7) and condition (A) it follows that,

$$
\operatorname{Max}\left(\|\tilde{\theta}\|_{r+1},\|\theta\|_{r+1}\right) \leq \hat{M} / \lambda^{r+1} .
$$

where $\hat{M}$ is some constant depending only on $\tilde{M}$ and constant $C$ (in condition A). Thus $\hat{M}$ depend only on $r, \gamma^{\prime}$ (hence on $\varepsilon$ ), function $f$, map $T$ and the manifold $\Omega$. 
Set $\psi=h \circ \theta$, we will show that $\psi$ is the required map. Clearly $\psi$ is of class $C^{r+1}$. First we will verify that $D_{r}\left(1^{\psi}, 1\right)<\delta$, to see this we recall the following general fact.

Given a manifold $\Omega$, a Lie group $G$ and $C^{r}$ maps, $h: \mathbb{R} \rightarrow G$ and $h_{i}: \Omega \rightarrow G$ and $g, g_{i}: \Omega \rightarrow \mathbb{R}(i=1,2)$, we have

$$
D_{r}\left(h \circ g_{1}, h \circ g_{2}\right) \leq L_{1}\left\|g_{1}-g_{2}\right\|_{r}
$$

and

$$
D_{r}\left(h_{1} \cdot[h \circ g]^{-1}, h_{2} \cdot[h \circ g]^{-1}\right) \leq L_{2} D_{r}\left(h_{1}, h_{2}\right)\|g\|_{r},
$$

where denotes the group multiplication. Note that constant $L_{1}$ depends only on the function $h$ and on the number of terms one gets in computing $r$ derivatives of composition of two functions by product and chain rule. Thus $L_{1}$ (and similarly $L_{2}$ ) depends only on $r$ and $h$. Thus we get,

$$
\begin{aligned}
D_{r}\left(1^{\psi}, 1\right) & =D_{r}\left((\psi \circ T) \psi^{-1}, 1\right)=D_{r}\left((\psi \circ T) \psi^{-1}, \psi \psi^{-1}\right) \\
& =D_{r}\left((\psi \circ T) \cdot(h \circ \theta)^{-1}, \psi \cdot(h \circ \theta)^{-1}\right) \leq L_{2} D_{r}(\psi \circ T, \psi)\|\theta\|_{r},
\end{aligned}
$$

(by (ii) where $L_{2}$ depends only on $r$ and $h$ ).

Now

$$
D_{r}(\psi \circ T, \psi)=D_{r}(h \circ(\theta \circ T), h \circ \theta) \leq L_{1}\|\theta \circ T-\theta\|_{r}, \quad \text { (by (i)). }
$$

Thus

$$
D_{r}\left(1^{\psi}, 1\right) \leq L\|\theta \circ T-\theta\|_{r}\|\theta\|_{r},
$$

where $L=L_{1} L_{2}-a$ constant depending only on $r$ and $h$.

Here we remark that if $G=\mathbb{R}$, then

$$
D_{r}\left(1^{\psi}, 1\right)=D_{r}(h \circ(\theta \circ T), h \circ \theta) \leq L\|\theta \circ T-\theta\|_{r},
$$

(without loss of generality $L>L_{1}$ ). Now consider,

$$
\begin{aligned}
\|\theta \circ T-\theta\|_{r} & =\frac{1}{q_{N}}\left\|\sum_{i=0}^{q_{N}-1} \tilde{\theta} \circ T^{i+1}-\tilde{\theta} \circ T^{i}\right\|_{r}=\frac{1}{q_{N}}\left\|\tilde{\theta} \circ T^{q_{N}}-\tilde{\theta}\right\|_{r} \\
& \leq \frac{K\|\tilde{\theta}\|_{r+1}}{q_{N}} a\left(q_{N}\right) \leq \frac{K \hat{M}}{\lambda^{r+1}} \frac{a\left(q_{N}\right)}{\left(q_{N}\right)} \quad \text { by }\left(7^{\prime}\right) .
\end{aligned}
$$

Now

$$
\lambda=\mu(V) \geq \mu(E) \geq \frac{1-\gamma^{\prime}}{q_{N}^{2}} \geq \frac{1}{2 q_{N}^{2}} \quad\left(\text { since } \gamma^{\prime}<\frac{1}{2}\right)
$$

Hence

$$
\|\theta \circ T-\theta\|_{r} \leq \frac{K \hat{M}}{q_{N}}\left(2 q_{N}^{2}\right)^{(r+1)} \cdot a\left(q_{N}\right)=K \hat{M}(2)^{r+1}\left(q_{N}\right)^{2 r+1} a\left(q_{N}\right) .
$$

This shows (using (8) and $\left(7^{\prime}\right)$ ) that,

$$
\begin{aligned}
D_{r}\left(1^{\psi}, 1\right) & \leq \frac{L K(\hat{M})^{2}(2)^{r+1}\left(q_{N}\right)^{2 r+1} a\left(q_{N}\right)}{\lambda^{r}} \leq L K(\hat{M})^{2}(2)^{2 r+1}\left(q_{N}\right)^{4 r+1} a\left(q_{N}\right) \\
& \leq \frac{L K(\hat{M})^{2}(2)^{2 r+1}}{\left(q_{N}\right)^{\rho}}<\delta \quad(\text { by }(2)) .
\end{aligned}
$$


Note that when $G=\mathbb{R},\left(8^{\prime}\right)$ shows that we need only assume $a(n)=1 / n^{2 r+1+\rho}$. Similarly in case of condition (B) we need only have $a(n)=1 / n^{r+1+\rho}$.

Now we prove that $\left\|\int_{\Omega} f(\psi(w) z) d \mu\right\|<\varepsilon$. Note that,

$$
\begin{aligned}
& \left|\int_{\Omega} f(\psi(w) z) d \mu-\sum_{i=0}^{\left(q_{N}\right)^{2}-q_{N}-1} \int_{T^{i} E} f(\psi(w) z) d \mu\right| \\
& \quad \leq M \mu\left(\Omega-\bigcup_{i=0}^{\left(q_{N}\right)^{2}-1} T^{i} E\right)+M \mu(E) q_{N} \\
& \left.\quad \leq \gamma^{\prime}+\frac{M}{q_{N}}, \quad \forall z, \text { (since } \mu(E)<1 /\left(q_{N}\right)^{2}\right) .
\end{aligned}
$$

Now we calculate $\int_{T^{i} E} f(\psi(w) z) d \mu$. Fix an $i \in\left\{0,1, \ldots,\left(q_{N}\right)^{2}-q_{N}-1\right\}$, and let $\tilde{w} \in T^{i} E$, say $\tilde{w}=T^{i} w, w \in E$. Because of the way $\theta$ was defined, we have $\theta(\tilde{w})=$ $\tilde{\theta}(\tilde{w})=\theta^{*}(w)$, thus

$$
\begin{aligned}
\int_{T^{i} E} f(\psi(\tilde{w}) z) d \mu & =\int_{T^{i} E} f([h \circ \theta] z) d \mu=\int_{E} f\left(\left[h \circ \theta^{*}\right] z\right) d \mu \\
& =\sum_{j=1}^{p} \int_{B_{i}} f\left(\left[h \circ \theta^{*}\right](z) d \mu .\right.
\end{aligned}
$$

Writing $A \stackrel{\gamma}{\sim} B$ to mean $|A-B|<\gamma$, we note that

$$
\sum_{j=1}^{p} \int_{B_{j}} f\left(\left[h \circ \theta^{*}\right] z\right) d \mu \stackrel{\left(\gamma^{\prime} /\left(q_{N}\right)^{2}\right)}{\sim} \sum_{j=1}^{p} \int_{V_{j}} f\left(\left[h \circ \theta^{*}\right] z\right) d \mu \quad(\text { by }(4)) .
$$

Now since $\left(\phi_{\alpha(j)}\right) *\left(\left.\mu\right|_{0_{\alpha}}\right)=\left.m\right|_{Q}$, we have

$$
\begin{array}{cc}
\int_{V_{i}} f\left(\left[h \circ \theta^{*}\right] z\right) d \mu=\frac{\mu\left(V_{j}\right)}{m\left(S_{j}\right)} \int_{S_{j}} f\left(\left[h \circ \eta_{j}(r)\right] z\right) d m(r) \quad \text { where } S_{j}=\phi_{\alpha(j)}\left(V_{j}\right) . \\
\underset{\left(\mu\left(V_{j}\right) \gamma^{\prime}\right)}{\sim} \frac{\lambda \mu\left(V_{j}\right)}{\lambda_{j}} \int_{I_{j}} f(h(t) z) d t=\lambda \int_{I_{i}} f(h(t) z) d t, \quad(\text { by }(6)) . \\
\sim\left(\lambda_{j} \gamma^{\prime}\right) / \lambda\left(q_{N}\right)^{2} \\
\sim(E) \int_{I_{j}} f(h(t) z) d t, \quad\left(\text { since } \lambda=\mu(V),\left|I_{j}\right|=\lambda_{j} / \lambda \quad\right. \text { and } \\
\mu(V-E)<\left(\gamma^{\prime} /\left(q_{N}\right)^{2} M\right) \quad(\text { by }(4)) .
\end{array}
$$

Thus

$$
\begin{aligned}
& \left|\sum_{j=1}^{p} \int_{B_{j}} f\left(\left[h \circ \theta^{*}\right] z\right) d \mu-\mu(E) \int_{0}^{1} f(h(t) z) d t\right| \\
& \quad \leq \gamma^{\prime} /\left(q_{N}\right)^{2}+\sum_{j=1}^{p}\left(\gamma^{\prime} \lambda_{j}\right)+\left(\gamma^{\prime} \lambda_{j} / \lambda\left(q_{N}\right)^{2}\right) \quad\left(\text { since } \lambda_{j}=\mu\left(V_{j}\right)\right) \\
& \quad \leq \gamma^{\prime} \lambda+2 \gamma^{\prime} /\left(q_{N}\right)^{2} \quad\left(\text { since } \sum_{j=1}^{p} \lambda_{j}=\lambda\right) \\
& \quad \leq 3 \gamma^{\prime} /\left(q_{N}\right)^{2} .
\end{aligned}
$$


Thus (9) and (10) gives us,

$$
\begin{aligned}
\left|\int_{\Omega} f(\psi(w) z) d \mu\right| \leq & \left(\gamma^{\prime}+\frac{M}{q_{N}}\right)+\left[\left(q_{N}^{2}\right)-q_{N}-1\right] \frac{3 \gamma^{\prime}}{\left(q_{N}\right)^{2}} \\
& +\left(\left(q_{N}\right)^{2}-q_{N}-1\right) \mu(E)\left|\int_{0}^{1} f(h(t) z) d t\right| \\
\leq & 4 \gamma^{\prime}+\frac{M}{q_{N}}+\left|\int_{0}^{1} f(h(t) z) d t\right|, \quad\left(\text { since } \mu(E)<1 /\left(q_{N}\right)^{2}\right) \\
\leq & 4 \gamma^{\prime}+(\varepsilon / 10)+\left|\int_{0}^{1} f(h(t) z) d t\right|, \quad \forall z \in Z \quad \text { by }(2) .
\end{aligned}
$$

Thus by (1) we get,

$$
\left.\left\|\int_{\Omega} f(\psi(w) z) d \mu\right\| \leq 4 \gamma^{\prime}+\varepsilon / 10+\varepsilon / 2<\varepsilon \quad \text { (if we choose } \gamma^{\prime}<\varepsilon / 10\right)
$$

The general case: now let $f \in C_{0}(Z \times \Omega)$ be any function. Set $g(z)=\int_{\Omega} f(z, w) d \mu(w)$. Now given $g, \varepsilon, \delta$, we will apply the previous technique to construct the function $\psi$ such that,

$$
D_{r}\left(1^{\psi}, 1\right)<\delta \quad \text { and } \quad\left\|\int_{\Omega} g(\psi(w) z) d \mu(w)\right\|<\varepsilon / 5 .
$$

In constructing this $\psi$ we will be a little more careful in choosing $N \in \mathbb{N}$, (see (2)). Here we pick $N \in \mathbb{N}$ so that in addition to conditions in (2) we also have,

$$
\left\|\frac{1}{q_{N}} \sum_{i=0}^{q_{N}-1} f\left(z, T^{-i} w\right)-g(z)\right\|<\varepsilon / 5
$$

(This is possible by the Ergodic theorem.) Set

$$
f_{1}(z, w)=\frac{1}{q_{N}} \sum_{i=0}^{q_{i}-1} f\left(z, T^{-i} w\right), \quad \text { then } \quad\left\|f_{1}-g\right\|<\varepsilon / 5 .
$$

Now we claim that,

$$
\left\|\int_{\Omega} f_{1}(\psi(w) z, w) d \mu-\int_{\Omega} f(\psi(w) z, w) d \mu\right\|<2 \varepsilon / 5 .
$$

To see this note that, if $w \in \Omega^{\prime}=\bigcup\left\{T^{i} E \mid 0 \leq i \leq\left(q_{N}\right)^{2}-q_{N}-1\right\}$ then $\psi\left(T^{i} w\right)=\psi(w)$ $\forall 0 \leq i \leq q_{N}-1$, (see the definition of $\psi$ ).

Thus

$$
\begin{aligned}
& \left|\int_{\Omega} f_{1}(\psi(w) z, w) d \mu-\int_{\Omega} f(\psi(w) z, w) d \mu\right| \\
& \quad=\left|\frac{1}{q_{N}} \sum_{i=0}^{q^{-1}}\left[\int_{\Omega} f\left(\psi(w) z, T^{-i} w\right) d \mu-\int_{\Omega} f(\psi(w) z, w) d \mu\right]\right| \\
& \quad=\left|\frac{1}{q_{N}} \sum_{i=0}^{q_{V}^{-1}}\left[\int_{\Omega} f\left(\psi\left(T^{i} w\right) z, w\right) d \mu-\int_{\Omega} f(\psi(w) z, w) d \mu\right]\right| \\
& \quad \leq \mid \frac{1}{q_{N}} \sum_{i=0}^{q_{V}^{-1}}\left[2 M \mu\left(\Omega-\Omega^{\prime}\right)\right] \leq 2 M\left(\frac{\gamma^{\prime}}{M}+\frac{1}{q_{N}}\right)<\frac{2 \varepsilon}{5} \quad \text { (since } \gamma^{\prime}<\varepsilon / 10(\text { and by }(2)) .
\end{aligned}
$$

Thus combining (a), (b) and (c) we get $\left\|\int_{\Omega} f(\psi(w) z, w) d \mu\right\|<\varepsilon$. 
Note that if $a(n)=1 / a^{n}$, it is easy to see that $D_{r}\left(1^{\psi} \psi\right)<\delta$ for any given $r \in \mathbb{N}$, (see the estimates). This takes care of part (III) of theorem (2.2).

Now we turn to part (II) of theorem (2.2). Note that condition (A) was used only to establish $D_{r}\left(1^{\psi}, 1\right)<\delta$. To make $\|\theta \circ T-\theta\|_{r}$ small, we used the technique of defining $\theta$ as an average of map $\tilde{\theta}$ and subsequently dominated $\|\theta \circ T-\theta\|_{r}$ by $\|\tilde{\theta}\|_{r+1}$. Condition (A) was then used to bound $\|\tilde{\theta}\|_{r+1}$ by $\left\|\theta^{*}\right\|_{r+1}$. Now if we set $\hat{\theta}(\omega)=\sum_{i=0}^{q_{\star}{ }^{-1}} \theta^{*}\left(T^{i} \omega\right)$ and $\psi=h \circ \hat{\theta}$, then $\|\hat{\theta} \circ T-\theta\|_{r}$ gets dominated directly by $\left\|\theta^{*}\right\|_{r+1}$ and thus we avoid using condition (A).

Since $\hat{\theta}$ is not the average of $\theta^{*}$, we have another problem namely in showing that $\left\|\int_{\Omega} f(\psi(w) z) d \mu\right\|<\varepsilon$. Since $\operatorname{supp} \theta^{*} \subseteq V$ and $\left\{T^{i} V \mid 0 \leq i \leq\left(q_{N}\right)^{2}-1\right\}$ are disjoint, $\hat{\theta}$ will be zero on 'most of $\Omega$ '. To overcome this problem we take Rokhlin tower of height $\left(q_{N}\right)$ (rather than $\left.\left(q_{N}\right)^{2}\right)$ over set $E$. Then exactly as before, it follows that $\int_{E} f(\psi(w) z) d \mu$ is close to $\mu(E) \int_{0}^{1} f(h(t) z) d t$ uniformly in $z$.

Now if $\tilde{w} \in T^{i} E$, say $\tilde{w}=T^{i} w, w \in E,\left(0 \leq i \leq q_{N}-1\right)$ then note that,

$$
\begin{aligned}
|\hat{\theta}(\tilde{w})-\hat{\theta}(w)| & =\left|\sum_{j=0}^{i-1} \theta^{*}\left(T^{q_{N}+j} w\right)-\sum_{j=0}^{i-1} \theta^{*}\left(T^{j} w\right)\right| \\
& \leqq K \sum_{j=0}^{i-1}\left\|\theta^{*} \circ T^{j}\right\|_{v} a\left(q_{N}\right) \leqq K q_{N} a\left(q_{N}\right),
\end{aligned}
$$

(this follows from condition (B)). Thus for large enough $N$ this is small. This shows that, $\int_{T^{i} E} f(\psi(w) z) d \mu$ is close to $\mu(E) \int_{0}^{1} f(h(t) z) d t, \forall 0 \leq i \leq q_{N}-1$. As before this enables us to show that $\left\|\int_{\Omega} f(\psi(\omega) z) d \mu\right\|<\varepsilon$.

Since we now have a Rokhlin tower of height $q_{N},\|\hat{\theta} \circ T-\hat{\theta}\|_{r}$ is of the order of $\left(q_{N}\right)^{r+1}$ and $\|\theta\|_{r}$ is of the order of $\left(q_{N}\right)^{r}$. Notice that now since $\hat{\theta}$ is not the average of $\theta^{*}$ we will be missing a ' $q_{N}$ ' in the denominator. This shows that to make $D_{r}\left(1^{\psi}, 1\right)<\delta$, one needs $a(n)$ to be of the order of $1 / n^{2 r+1+\rho}$.

Finally, let us consider part (IV) of theorem (2.2). First note that since $G=\mathbb{R}$, by (II) we already have the result if $\left|\alpha-p_{n} / q_{n}\right|<k / q_{n}^{2+r+o}$, but in this example we actually have more than $C^{r}$ rigidity. Namely the transformation $T$ itself can be approximated in $C^{r}$ norm by periodic transformation $T_{n},\left(T_{n}\right.$ being the rotation by $p_{n}\left(q_{n}\right)$. This enables us to partition the circle into $q_{N}$ arcs of equal lengths and define $\theta$ suitably on one of the arcs and extend it periodically on all of the circle. Hence we can write,

$$
\|\theta \circ T-\theta\|_{r}=\left\|\theta \circ T-\theta \circ T_{n}\right\|_{r} \leq(\text { Constant })\|\theta\|_{r+1}\left(1 / q_{n}^{r+1+\rho}\right)
$$

by the Mean Value Theorem. Since $\|\theta\|_{r+1}$ is of the order $q_{N}^{r+1}$ we can make $\|\theta \circ T-\theta\|_{r}$ arbitrarily small. (See [19] for details in this case.)

Now we give the proof of the following fact used in (1).

Proposition. 3 [6]. Assume that $(Z, G, \nu)$ is an ergodic dynamical system with the $L^{\infty}$-fixed point property. Then given $f \in L^{1}(Z, \nu)$ with $\int f d \nu=0$ and $\varepsilon>0, \exists c_{i} \varepsilon[0,1]$ and $g_{i} \in G, 1 \leq i \leq s$ such that $\sum_{i=1}^{s} c_{i}=1$ and $\left\|\sum_{i=1}^{s} c_{i} f_{g_{i}}\right\|<\varepsilon_{\text {. }}=$

Proof. Let $C(f)$ be the closed convex hull of $\left\{f_{g} \mid g \in G\right\}$ in $L^{1}(Z, \nu)$. Let dist. $(0, C(f))=\delta$. If $\delta>0$ then by the Hahn-Banach theorem $\exists f^{*} \in L^{\infty}(Z, \nu)$, with $\left\|f^{*}\right\|_{\infty}=1$ such that $\int h f^{*} d \nu \geq \delta / 2 \forall h \in C(f)$. 
Let $C^{*}\left(f^{*}\right)$ be the weakly closed convex hull of $\left\{f_{g}^{*} \mid g \in G\right\}$ in $L^{\infty}(Z, \nu)$. Note that $\int f h^{*} \geq \delta / 2 \forall h^{*} \in C^{*}\left(f^{*}\right)$. Now since $C^{*}\left(f^{*}\right)$ is $G$-invariant, convex and weakly compact, by the $L^{\infty}$-fixed point property there exists a $G$-invariant function say $C$ in $C^{*}\left(f^{*}\right)$, but then ergodicity implies that almost surely $C$ is a constant. Thus, since $C \in C^{*}\left(f^{*}\right)$, we have $\delta / 2 \leq \int C f=C \int f=0$, a contradiction. Hence $\delta=0$ and this proves the proposition.

We remark that the function $f$ to which we have applied this proposition may not have zero integral, however we have chosen this $f$ such that $\|f\|<\varepsilon / 8$, (see early part of $\S 2$ ) and it is easy to see that this is enough to ensure $\left\|\sum c_{i} f_{g_{1}}\right\|<\varepsilon / 4$. Proof of sublemma 2. First let $W$ be the cube $\left\{\bar{x}=\left(x_{1}, \ldots, x_{n}\right) / 0<x_{i}<a\right\}$ and $I=[0, \lambda]$. Let $\chi: W \rightarrow[0,1]$ be a $C^{\infty}$ map such that $\chi \equiv 0$ outside $\left\{\bar{x} \mid 1 / 2 \varepsilon<x_{i}<a-\right.$ $1 / 2 \varepsilon\}$ and $\chi \equiv 1$ inside $\left\{\bar{x} \mid 1 / \varepsilon<x_{i}<a_{i}-1 / \varepsilon\right\}$ and $\|\chi\|_{r} \leq M(r)(\varepsilon)^{r}, \forall 1 \leq i \leq r$ where $\varepsilon=(a / N), N \in \mathbb{N}$ and $M(r)$ is some constant depending on $r$ alone. Explicit construction of such a function can be found in [21] and is based on the usual convolution technique. Take $g(\bar{x})=g\left(x_{1}, \ldots, x_{n}\right)=\left(\lambda x_{n} / a\right)$ and set $\eta(\bar{x})=$ $\chi(\bar{x}) g(\bar{x})$. It is easy to verify that by choosing $N \in \mathbb{N}$ large enough one can make $[1 / m(W)] \int_{W} f \circ \eta d m$ arbitrarily close to $(1 / \lambda) \int_{0}^{\lambda} f(t) d t$ and this can be done uniformly over the class of functions which are uniformly bounded (in particular for an equicontinuous family). If $W$ is not a cube then write 'most of $W$ ' as a disjoint union of such $p$ cubes (or its translates) and apply above result replacing $\lambda$ by $\lambda / p$ and interval of length $\lambda$ by intervals of length $\lambda / p$ and take the sum of the corresponding functions.

Now we very briefly indicate the proof of theorem 2.5. See [19] for the details in the case of a 2-torus where the underlying construction of the required function is much easier.

The techniques used are as before. We will only sketch the ideas involved in lifting proximality generically, (see $[4,19]$ ). Fix $w_{0} \in \Omega$. Given open sets $U, V \subseteq \Sigma$ and $\varepsilon>0$, define

$$
\begin{aligned}
P(U, V, \varepsilon)= & \left\{X_{A} \in \bar{B}_{\infty} \mid \operatorname{diam}\left(H_{t}\left(X_{A}\right)\left(U x\left\{w_{0}\right\}\right) \cup\left(H_{t}\left(X_{A}\right)\left(V x\left\{w_{0}\right\}\right)\right)\right.\right. \\
& <\varepsilon, \text { for some } t \in \mathbb{R}\},
\end{aligned}
$$

where $H_{t}\left(X_{A}\right)$ is the homeomorphism given by $H_{t}\left(X_{A}\right)([x], w)=\left(\left[X_{A}(w, t) x\right], w \cdot t\right)$. If $X_{A} \in P(U, V, 1 / n), \forall n$ and $\forall U, V$ belonging to a countable base of $\sum$, then any pair of points in the fiber over $w_{0}$ is proximal and since $(\Omega, \mathbb{R})$ is minimal, this implies $\dot{x}=A(w \cdot t) x$ is a proximal system. Thus as before the main problem is to prove that $P(U, V, \varepsilon)$ is dense in $\bar{B}_{\infty}$. Using the same arguments as in the proof of theorem (2.2) we reduce this problem to showing that $1 \in \overline{P(U, V, \varepsilon)}$ and this is equivalent to showing that given a $g_{0} \in S L(n, \mathbb{R})$ and $\delta>0, \exists \psi C^{\infty}(\Omega, S L(n, \mathbb{R}))$ such that $D_{\infty}\left(1^{\psi}, 1\right)<\delta$ and $\psi\left(w_{0}\right)=g_{0}$, (see [19] for details). Construction of such a function is essentially as before, in fact much easier, (see [19]).

In the same way, under the same assumptions one can show residuality of recurrent as well as uniquely ergodic systems in $\bar{B}_{\infty}$, (see $[4,19]$ ).

Remark 4. The (Sacker-Sell) spectrum of system (I) is defined to be the set $\sigma(A)=$ $\{\lambda \mid \lambda \in \mathbb{R}$ such that for some $w \in \Omega$, the equation $\dot{x}=[A(w \cdot t)-\lambda I] x$ has a non-trivial 
bounded solution\}. The spectral theorem of Sacker and Sell [20] says that $\sigma(A)=$ $\bigcup_{i=1}^{k}\left[a_{i}, b_{i}\right]$, where $k \leq n$ and the union is a disjoint union of $k$-intervals. If $a_{i}=b_{i} \forall 1 \leq i \leq k$ then system (I) is said to have a discrete spectrum otherwise it is said to have a band spectrum. If the fiow on $\Omega$ is periodic then the spectrum $\sigma(A)$, for any $\boldsymbol{A}$ is discrete.

Following R. Johnson, we will call system (I) elliptic iff $\int_{\Sigma} H d \nu=0$ for all Borel probability measures $\nu$ on $\sum$ which are invariant under the skew-product flow, where $H([x], w)=\langle A(w) x, x\rangle /\|x\|^{2}$ and $<,>$ is the standard inner product on $\mathbb{R}^{n}$. In the case $n=2$, if system (I) is not elliptic it is said to be hyperbolic, (see [9] for details).

The following proposition is proved in [9] and [10] for $n=2$.

Proposition 5. (a) If the skew-product flow on $\sum$ is uniquely ergodic then system (I) is elliptic. (b) If system (I) is hyperbolic then it is proximal if and only if it has a band spectrum.

Our result shows that for flows admitting fast-periodic approximation, smooth elliptic, recurrent proximal cocycles are generic in the class $\bar{B}_{\infty}$. Now note that for the rotation flow on $\mathbb{T}^{2}$ generated by badly approximable irrationals, smooth elliptic proximal cocycles do exist, namely take any smooth enough elliptic cocycle in the subgroup of uppertriangular matrices. Then by standard Kolmogorov-Siegal argument one can show that it is cohomologous to a cocycle of the form

$$
\left(\begin{array}{cc}
1 & b t \\
0 & 1
\end{array}\right)
$$

and thus cannot yield a minimal skew-product. Hence a more appropriate question is: can one have smooth elliptic recurrent-proximal cocycles based on such rotation flows (i.e. flows generated by badly approximable irrationals). We conjecture that this is not possible if $\boldsymbol{A}$ is smooth enough.

Finally, smooth cocycles which are hyperbolic and proximal have very complicated dynamical properties and their construction for $\mathbb{R}$ and $\mathbb{Z}$ actions is the content of [17] and [7] respectively. In the situation when $\Omega=\mathbb{T}^{2}$ - the 2 torus, the cocycle is hyperbolic with discrete spectrum and is smooth enough, using the usual small divisor argument Johnson and Sell ([11]) have shown that such a cocycle must be cohomologous to a constant cocycle, (i.e. the cocycle of the form $e^{i K}$ for some fixed matrix $K$ ). In general the questions regarding proximality or band spectrum of linear systems $\dot{x}=A(w \cdot t) x$ are related to the nature of the Mackey range and the recurrence and transience properties of $X_{A}$. However no concrete results of this nature are available. Much more is known about some specific cocycles, in particular those arising as the fundamental matrix solutions of the 1-dimensional Schrödinger equation, (see [8], an exhaustive list of other references is too long and hence will be omitted).

\section{Acknowledgement}

The author wishes to thank the referee for his comments and suggestions which improved the original version. 


\section{REFERENCES}

[1] D. Anosov \& A. Katok. New examples in smooth ergodic theory. Trans. Moscow Math. Soc. 23 (1970), 1-35.

[2] M. Brin. Topological transitivity of one class of dynamical systems and flows of frames on manifolds of negative curvature. Funct. Anal. 9 No. 1 (1975), 8-16.

[3] R. Ellis \& R. Johnson. Topological dynamics and linear differential systems. J. Diff. Equations 44 No. 1 (1982), 21-39.

[4] S. Glasner \& B. Weiss. On the construction of minimal skew products. Israel J. Math. 34 No. 4 (1979).

[5] J. Hawkins. Smooth $\mathbb{T}^{n}$ valued cocycles for ergodic diffeomorphisms. Proceedings of AMS 93 No. 2 (1985), 307-311.

[6] M. Herman. Construction de diffeomorphismes ergodiques (1979) preprint.

[7] M. Herman. Construction d'un diffeomorphisme minimal d'entropie topologique non nulle. Ergod. Th. \& Dynan. Syst. 1 (1981), 65-76.

[8] M. Herman. Une Methode pour minorer les exposonts de Lyapounov. Comment. Math. Helv. 58 (1983), 453-502.

[9] R. Johnson. Ergodic theory and linear differential equations. J. Diff. Equations 28 (1978), 23-34.

[10] R. Johnson. On Floquet theory for almost periodic 2-dimensional linear systems. J. Diff. Equations 37 (1980).

[11] R. Johnson \& G. Sell. Smoothness of spectral subbundles and reducibility of quasi-periodic linear differential systems. J. Diff. Equations 41 (1981), 262-288.

[12] A. Katok. Constructions in ergodic theory Vol. I and II. Lecture notes (to be published).

[13] A. Katok \& A. Stepin. Approximations in ergodic theory. Russian Math. Surveys 22 No. 5 (1967), 77-102.

[14] H. Keynes \& M. Nerurkar. Ergodicity in affine skew product toral extensions. Pacific J. Math. 123 No. 1 (1986).

[15] A. Krygin. Examples of ergodic cylindrical cascades. Math. Notes U.S.S.R. 16 (1974), 1180-1186.

[16] A. Livsic. Cohomology of dynamical systems. Math. U.S.S.R. Izvestija No. 6 (1972), 1278-1301.

[17] V. Millionschikov. Proof of the existence of irregular systems of linear differential equation with almost periodic coefficients. Differential Equations (translated from Russian) 4 No. 3 (1968).

[18] M. Nerurkar. Ergodic continuous skew product actions of amenable groups. Pacific J. Math. 119 No. 2 (1985).

[19] M. Nerurkar. Recurrent-proximal linear differential systems. Proc. AMS 100 No. 4 (1987) 739.

[20] R. Sacker \& G. Sell. A spectral theory for linear differential systems. J. Diff. Equations 27 (1978), 320-358.

[21] V. Vladimirov. Generalized functions in Mathematical Physics. (Mir Publisher).

[22] R. Zimmer. Amenable ergodic group actions and an application to Poisson boundaries of Random walks. J. Functional Analysis 27 No. 3 (1978), 350-372. 